\title{
Antibiotics Act with vB_AbaP_AGC01 Phage against Acinetobacter baumannii in Human Heat-Inactivated Plasma Blood and Galleria mellonella Models
}

\author{
Bartłomiej Grygorcewicz ${ }^{1, *(1)}$, Marta Roszak ${ }^{1}$, Piotr Golec ${ }^{2}{ }^{\mathbb{D}}$, Daria Śleboda-Taront ${ }^{1}$, \\ Natalia Łubowska ${ }^{3}{ }^{\circledR}$, Martyna Górska ${ }^{4}$, Joanna Jursa-Kulesza ${ }^{5}$, Rafał Rakoczy ${ }^{6}{ }^{\circledR}$, \\ Bartosz Wojciuk $^{7}$ and Barbara Dołęgowska ${ }^{1}$ \\ 1 Department of Laboratory Medicine, Pomeranian Medical University in Szczecin, Powstańców \\ Wielkopolskich 72, 70-111 Szczecin, Poland; martaroszak95@gmail.com (M.R.); \\ daria.sleboda@pum.edu.pl (D.Ś.-T.); barbara.dolegowska@pum.edu.pl (B.D.) \\ 2 Department of Molecular Virology, Institute of Microbiology, Faculty of Biology, University of Warsaw, \\ Miecznikowa 1, 02-096 Warsaw, Poland; pgolec@biol.uw.edu.pl \\ 3 Department of Medical Microbiology, Faculty of Medicine, Medical University of Gdansk; \\ Marii Skłodowskiej-Curie 3a, 80-210 Gdańsk, Poland; nlubowska@gumed.edu.pl \\ 4 Department of General Pathology, Pomeranian Medical University in Szczecin, \\ Powstańców Wielkopolskich 72, 70-111 Szczecin, Poland; martyna.gorska@pum.edu.pl \\ 5 Department of Medical Microbiology, Pomeranian Medical University in Szczecin, \\ Powstańców Wielkopolskich 72, 70-111 Szczecin, Poland; asiaju@pum.edu.pl \\ 6 Institute of Chemical Engineering and Environmental Protection Process, Faculty of Chemical Engineering, \\ West Pomeranian University of Technology, al. Piastów 42, 71-065 Szczecin, Poland; rrakoczy@zut.edu.pl \\ 7 Department of Diagnostic Immunology, Pomeranian Medical University in Szczecin, \\ Powstańców Wielkopolskich 72, 70-111 Szczecin, Poland; bartosz.wojciuk@pum.edu.pl \\ * Correspondence: bartlomiej.grygorcewicz@pum.edu.pl
}

Received: 27 April 2020; Accepted: 19 June 2020; Published: 19 June 2020

Abstract: Increasing multidrug resistance has led to renewed interest in phage-based therapy. A combination of the bacteriophages and antibiotics presents a promising approach enhancing the phage therapy effectiveness. First, phage candidates for therapy should be deeply characterized. Here we characterize the bacteriophage vB_AbaP_AGC01 that poses antibacterial activity against clinical Acinetobacter baumannii strains. Moreover, besides genomic and phenotypic analysis our study aims to analyze phage-antibiotic combination effectiveness with the use of ex vivo and in vivo models. The phage AGC01 efficiently adsorbs to A. baumannii cells and possesses a bacteriolytic lifecycle resulting in high production of progeny phages $\left(317 \pm 20 \mathrm{PFU} \times \mathrm{cell}^{-1}\right)$. The broad host range (50.27\%, 93 out of 185 strains) against A. baumannii isolates and the inability of AGC01 to infect other bacterial species show its high specificity. Genomic analysis revealed a high similarity of the AGC01 genome sequence with that of the Friunavirus genus from a subfamily of Autographivirinae. The AGC01 is able to significantly reduce the A. baumannii cell count in a human heat-inactivated plasma blood model (HIP-B), both alone and in combination with antibiotics (gentamicin (GEN), ciprofloxacin (CIP), and meropenem (MER)). The synergistic action was observed when a combination of phage treatment with CIP or MER was used. The antimicrobial activity of AGC01 and phage-antibiotic combinations was confirmed using an in vivo larva model. This study shows the greatest increase in survival of G. mellonella larvae when the combination of phage $(\mathrm{MOI}=1)$ and MER was used, which increased larval survival from $35 \%$ to $77 \%$. Hence, AGC01 represents a novel candidate for phage therapy. Additionally, our study suggests that phages and antibiotics can act synergistically for greater antimicrobial effect when used as combination therapy.

Keywords: Acinetobacter baumannii; antibiotics resistance; bacteriophages; blood infection; Galleria mellonella 


\section{Introduction}

The rising incidence of multi- and pan-drug resistant bacterial strains poses a severe challenge to medical care worldwide [1,2]. The majority of drug-resistant infection is associated with members of the ESKAPE (Enterococcus faecium, Staphylococcus aureus, Klebsiella pneumoniae, Acinetobacter baumannii, Pseudomonas aeruginosa, and Enterobacter species) group [3]. According to the WHO list of antibiotic-resistant bacteria, carbapenem-resistant $A$. baumannii poses a critical threat to public health, with limited therapeutic options. Ipso facto, the WHO has highlighted the need to prioritize research on the development of new antibiotics and alternative treatment options for the listed drug-resistant bacteria [4].

A. baumannii causes one of the most challenging nosocomial infections, with mortality rates as high as 35\% [5]. This bacterium is responsible for a large spectrum of hospital-acquired infections such as bacteremia, sepsis, urinary tract infection, pneumonia, and burn wound infections, and has developed resistance to almost all known antibiotics [6]. One of the most promising tools in combatting antibiotic-resistant bacteria is the use of bacteriophages, viruses that infect bacteria [7].

As a result of widespread antimicrobial drug resistance, alternative treatments such as bacteriophage therapy are gaining increasing interest. Bacteriophages specifically infect and propagate inside of bacterial cells and are promising tools to combat bacterial infection [8]. Phages appear to be safe and neutral to the human body and their application to healthy volunteers does not show any side effects [9]. Because of that, a lot of Acinetobacter baumannii phages were isolated in recent years. Unfortunately, a majority of these phages are poorly characterized and especially, a lack of in-depth genome exploration poses a problem for their therapeutic use $[10,11]$. Thus suggesting that isolation and characterization of novel lytic bacteriophages is urgently needed.

Here we fully characterize novel phage AGC01 (vB_AbaP_AGC01). Additionally, besides phenotypic and genomic characterization, we aimed to investigate the antimicrobial activity of AGC01 alone and in combination with antibiotics with the use of ex vivo and in vivo models. Enhancement of the phage activity by antibiotic addition was observed for e.g., Escherichia coli, Burkholderia cepacia, Pseudomonas aeruginosa [12,13]. Nevertheless, up to date only one A. baumannii infecting lytic phage KARL-1 was evaluated for antibiotic enhancement; result shows that MER augmented KARL-1 activity, but this study shows only in vitro-method based approach [11].

\section{Results}

\subsection{Bacteriophage Isolation and Host Range}

The isolated phage formed clear plaque surrounded by a halo zone (size: $1.6 \pm 0.3 \mathrm{~mm}$ in diameter). Over prolonged incubation the plaque size and halo zone increased to $4.6 \pm 0.4 \mathrm{~mm}$ (Figure 1A). The phage AGC01 was able to infect $50.27 \%$ (93 of 185) of the A. baumannii strains, including reference strains and clinical isolates (Table S1). Host range analysis showed that the AGC01 only infects A. baumannii and does not infect other species tested during this analysis (including Escherichia coli, Klebsiella spp., Enterobacter spp., and Pseudomonas spp. strains).

\subsection{Bacteriophage Growth and Stability Characterization}

The virulence of AGC01 phage was initially characterized in terms of growth and stability under various conditions. First, an adsorption analysis showed that approximately $99 \%$ of virions adsorb to host within $5 \mathrm{~min}$ (Figure 1B). The latent period of AGC01 was found to be $20 \mathrm{~min}$ long and the burst size comprised approximately $317 \pm 20$ progeny phages per infected cell (Figure 1C). Finally, the ability of the phage to lyse A. baumannii cells was evaluated. Complete clearance of the bacterial optical density was observed in A. baumannii liquid culture and attributed to efficient lysis of cells by AGC01 at all the multiplicities of infection (MOIs) used (Figure 1D). The AGC01 was therefore found to possess strong and concentration-dependent lytic activity against the strain used. 
The resistance of phage AGC01 to $\mathrm{pH}$ and temperature changes was investigated to assess the stability of the phage under various physicochemical conditions. Phage AGC01 remains active at $\mathrm{pH}$ values ranging from 5 to 7 , but loses activity at $\mathrm{pH} 3$. When incubated at $\mathrm{pH} 9$, within $2 \mathrm{~h}$ only $37 \%$ of virus particles remained active and able to infect the host, which further dropped to $10 \%$ active virus particles after exposure to $\mathrm{pH} 11$ (Figure 1E). Analysis of thermal stability of bacteriophage showed that AGC01 retains activity at $30{ }^{\circ} \mathrm{C}$ throughout the duration of this analysis, while time-dependent decreases in phage activity were observed at temperatures $40^{\circ} \mathrm{C}$ and $50^{\circ} \mathrm{C}$, and temperatures higher than $60^{\circ} \mathrm{C}$ resulted in immediate loss of phage activity (Figure 1F). Additionally, storage of AGC01 phage stock at $4{ }^{\circ} \mathrm{C}$ resulted in loss of only $13 \%$ of active virions after 14 months.

A
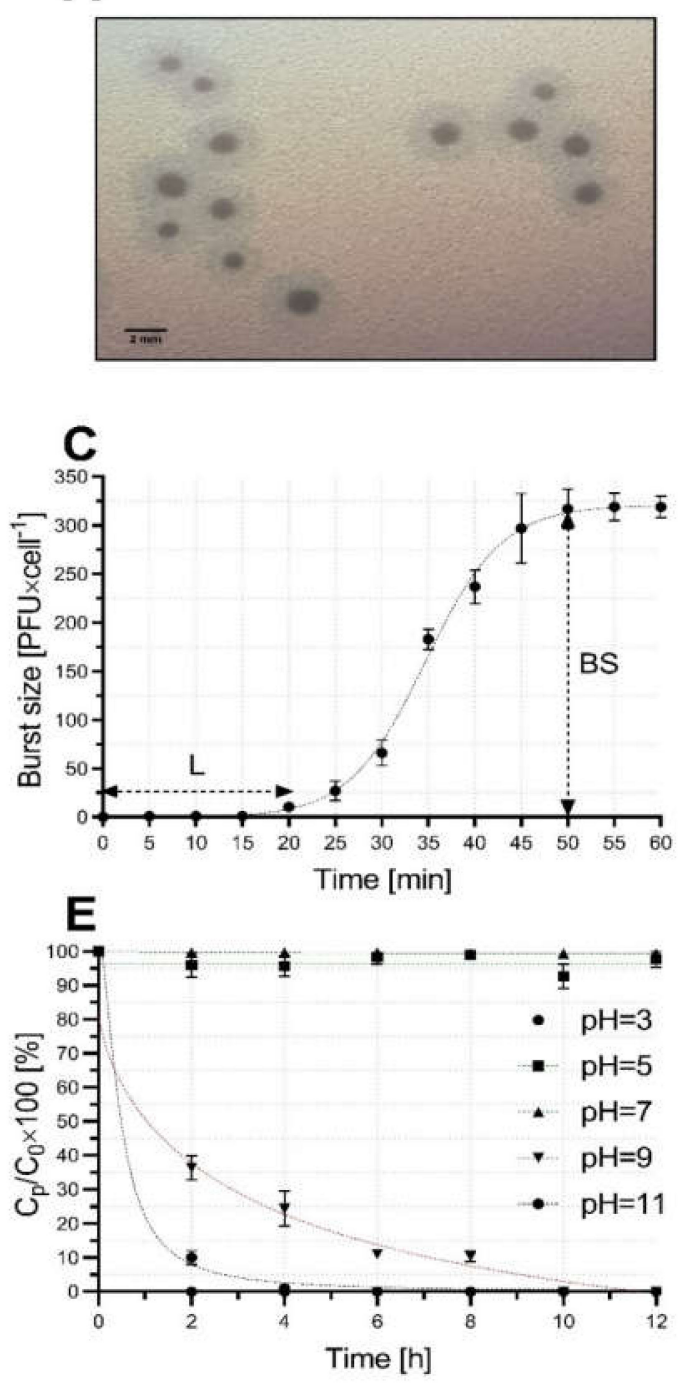

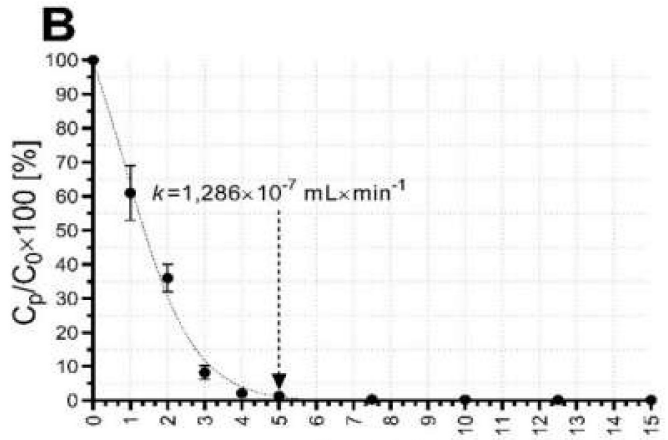

Time [min]

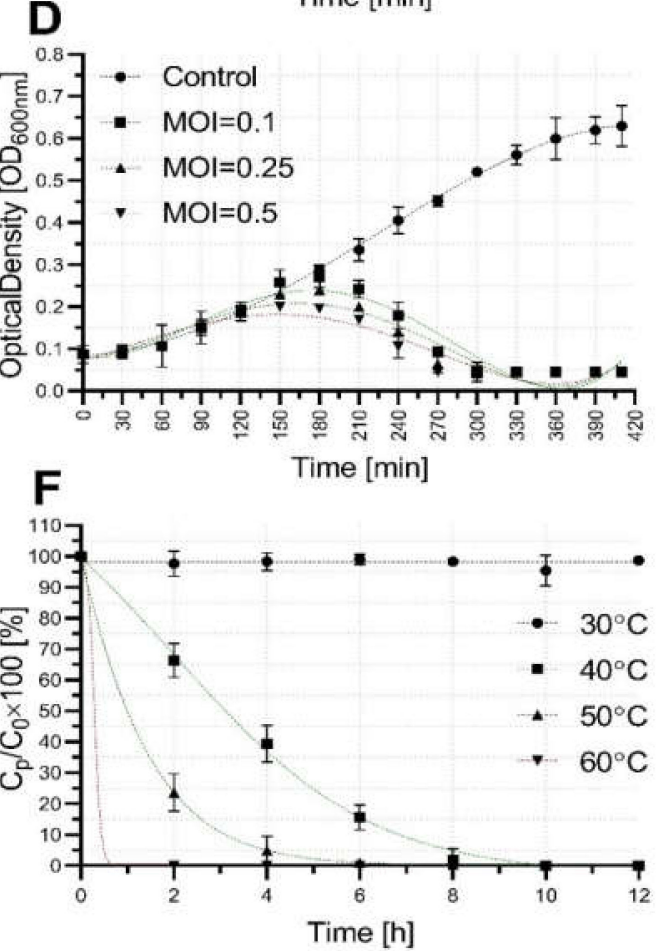

Figure 1. Characterization of bacteriophage vB_AbaP_AGC01 growth and stability. (A) Plaques formed by bacteriophage vb_AbaP_AGC01 after $18 \mathrm{~h}$ incubation at $37^{\circ} \mathrm{C}$. (B) Kinetics of the phage adsorption to host at a multiplicity of infection (MOI) of 0.1. (C) One-step growth curve indicating the latent period

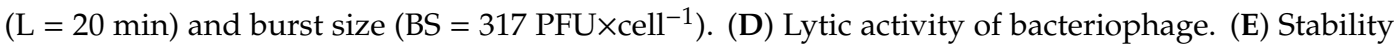
of bacteriophages at different $\mathrm{pH}$ values. All experiments were technically repeated three times with triplicate biological replication. (F) Susceptibility of isolated bacteriophage to increases in temperature. 


\subsection{Bacteriophage Comparative Genomics and Genome Analysis}

The AGC01 phage genome (dsDNA, length: 41,231 bp) has a G+C content (39.50\%) and a coding capacity (94.18\%) similar to other A. baumannii infecting phages [14]. Additionally, this $\mathrm{G}+\mathrm{C}$ content between $38.70 \%$ and $42.60 \%$ is similar to the $\mathrm{G}+\mathrm{C}$ content of $A$. baumannii host strains [15]. The genome structure of bacteriophage vB_AbaP_AGC01 features high similarity to other podoviruses from the Friunavirus genus in a subfamily of Autographivirinae. The highest similarity to the AGC01 genome was observed for Acinetobacter baumannii phage vB_AbaP_APK92 (acc. no. MK257721.1), with query coverage of about $96 \%$ and identity of $94.71 \%$. The sequence comparison between bacteriophages vB_AbaP_AS11, IME200, and FRI1 are presented in Figure 2C. RNA polymerase (RNAP)-based phylogenetic analysis confirmed the classification of the isolated bacteriophage as a Friunavirus genus member in a subfamily of Autographivirinae. The vB_AbaP_AGC01 phage was grouped in one clade together with Fri1 phage and vB_AbaP_AS11 (Figure 2D). Additionally, multiple genome alignments, showing conserved genomic sequence and rearrangements, of the selected $A$. baumannii infecting podoviruses from Autographivirinae are presented in Supplementary Figure S1.

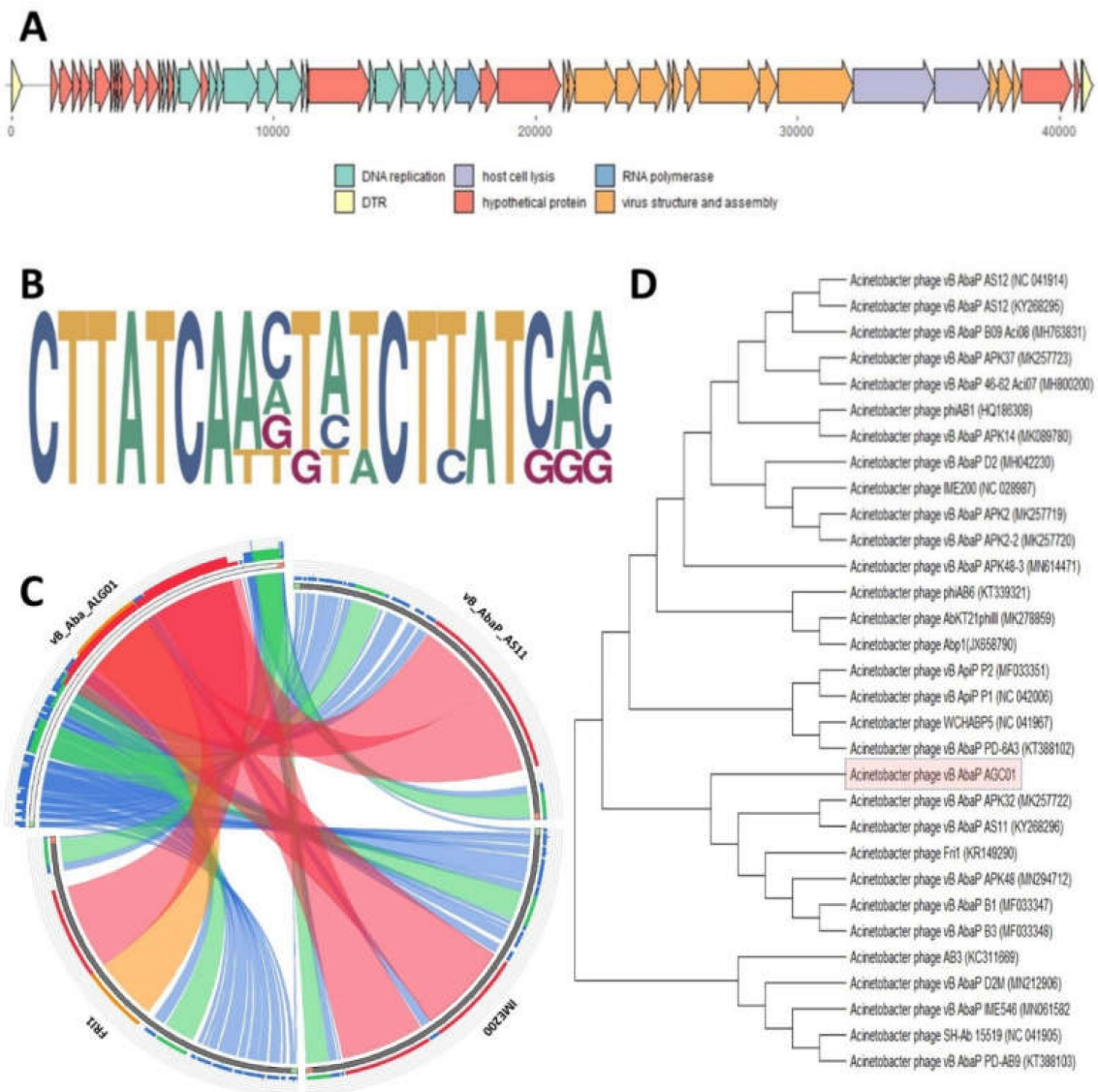

Figure 2. Genome organization, promoter structure, and comparative genomics of Acinetobacter phage vB_AbaP_AGC01. (A) Schematic presentation of the AGC01 genome organization. Arrows represent open reading frames (ORFs) identified in the genome and their transcriptional orientation. The coloring of genes represents the function of the genes: DNA replication (green), direct terminal repeat (DTR, yellow), virus structure and assembly (orange), host cell lysis (violet), hypothetical proteins (red). (B) Predicted putative phage promoter consensus of bacteriophage vb_AbaP_AGC01. (C) Circoletto visualization of the genome nucleotide sequence-structure similarity of the isolated phage with phages vB_AbaP_AS11 (acc. no. NC_041915.1), IME200 (acc. no. KT804908.2) and FRI1 (acc. no. KR149290.1). Ribbon colors correspond to similarity level (blue $\leq 0.25$; green $0.26-0.5$, orange $0.51-0.75$, red $>0.75$ ). (D) RNA polymerase-based phylogenetic tree of Acinetobacter infecting podoviruses based on RNA polymerase sequence. 
In total, 55 open reading frames (ORFs) were identified in the AGC01 genome, and the functions of 32 genes ( $58 \%$ ) were assigned as shown in Figure 2A and Supplementary Table S2. The genome is organized into functional modules that contain genes involved in DNA replication, RNAP, virus structure, and assembly, as well as host cell lysis (Supplementary Table S2). Moreover, the AGC01 lysis cassette, which encodes holin and endolysin, is spaninless, which strongly suggests that AGC01 uses the canonical holin-endolysin lysis pathway similar to that described for the phage Petty lysis system [16]. The regulatory gene promoters were identified and the putative phage promoter consensus (based on 7 identified promoters) is presented in Figure 2B.

Importantly, a lack of integrases, as well as other proteins involved in lysogeny, host conversion and toxins, supports the growth kinetics data, suggesting that AGC01 possesses lytic properties and could be used for therapeutic purposes.

\subsection{Activity of vB_AbaP_AGC01 on Biofilm, HIP-B, and G. Mellonella Larva Models}

To evaluate bacteriophage activity and suitability as an antibacterial agent, we first analyzed the antibiofilm activity of AGC01. The biofilm biomass of infection-susceptible A. baumannii was reduced to $71.57 \%$ and $84.76 \%(p<0.05)$ after incubation with AGC01 phage (Figure $3 \mathrm{~A})$. These data indicate that AGC01 possesses a high ability to reduce biofilm production.
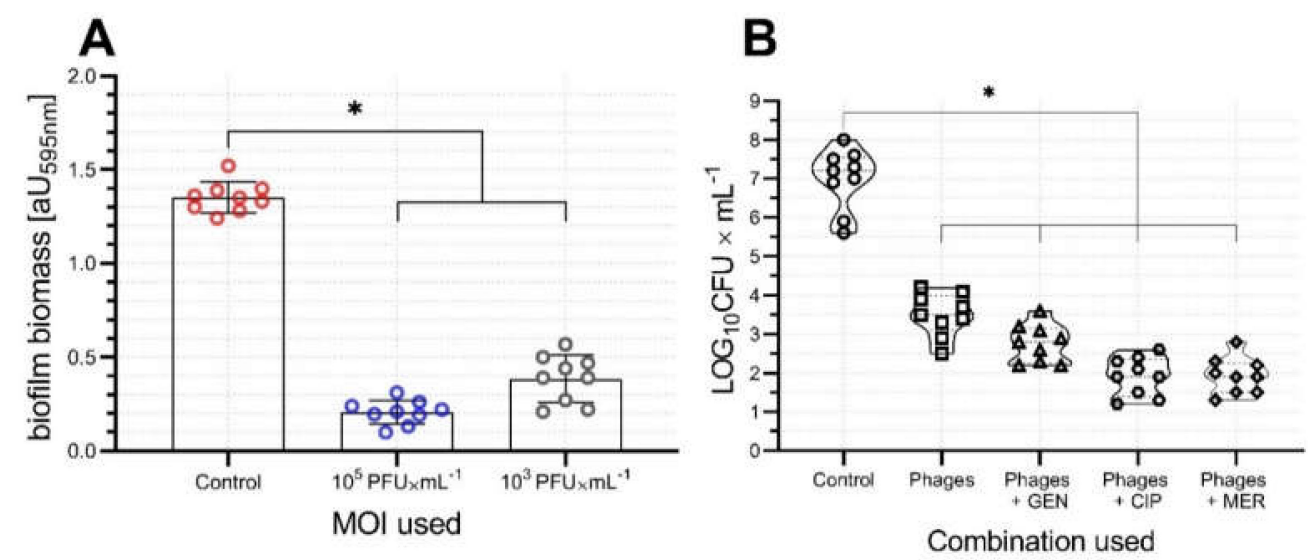

Figure 3. Activity of vB_AbaP_AGC01 with the use of the selected models. (A) Antibiofilm activity of isolated phage. (B) Antibacterial activity of AGC01 in human heat-inactivated plasma blood model alone and in combination with the antibiotics meropenem (MER), ciprofloxacin (CIP), and gentamicin $(\mathrm{GEN})$. The asterisk $\left(^{*}\right)$ indicates statistically significant data $(p<0.05)$. All experiments were technically repeated three times with triplicate biological replication.

To assess the ex vivo activity of AGC01 in blood, a heat-inactivated plasma blood model (HIP-B) was used. Phage AGC01 activity significantly reduced the MDRAB cell count when used alone and in combination with antibiotics in the HIP-B model (Figure 3B). The highest reduction of bacterial cell count was observed using phage in combination with CIP and MER, both of which resulted in an approximately $4 \log$ reduction ( $p<0.05$, compared to phage used alone). The poorest efficiency was observed using the phage and GEN combination, where only a slight reduction of bacterial cell count was achieved which was not found to be significant $(p=0.0667)$. The use of the antibiotic alone does not significantly influence the bacterial count compared to control conditions (Supplementary Figure S2A).

Using an in vivo G. mellonella larva model, reduced mortality was observed in larvae infected with $A$. baumannii when phage treatment was introduced. The highest increase in survival rate was observed using bacteriophage treatment at an MOI of 50, while lower MOIs (10 and 1) also increased the survival rate of infected larvae (Figure 4A). All MOIs resulted in a significant increase in larva survival when compared to untreated control $(p<0.05)$. 
Treatment with the antibiotics alone CIP, GEN, and MER did not notably improve survival of A. baumannii infected larvae, demonstrating the poor effectiveness of these antibiotics against MDRAB (Figure 4B). No statistically significant change in survival was observed for infected larvae treated with CIP $(p=0.08)$, MER, or GEN $(p=0.73)$. Treatment with MER resulted in a survival rate of $5 \%$ of larvae, while treatment with GER and CIP were not able to recover any larvae survival (all $p<0.05$ ). Additionally, larvae lethality or melanization was not observed after antibiotics alone injection.

The combination of the antibiotics MER and CIP with phage treatment improved survival of A. baumannii-infected G. mellonella larvae compared to the single phage treatment, as well as relative to larvae treated with phage only (Figure 4C). The highest increase in survival rate (from 35\% to 77\%) was observed in infected larvae treated with a combination of phage $(\mathrm{MOI}=10)$ and MER $(p<0.05)$. All combinations used had a significant increase of larva survival if compared to bacterial injected control $(p<0.05)$.
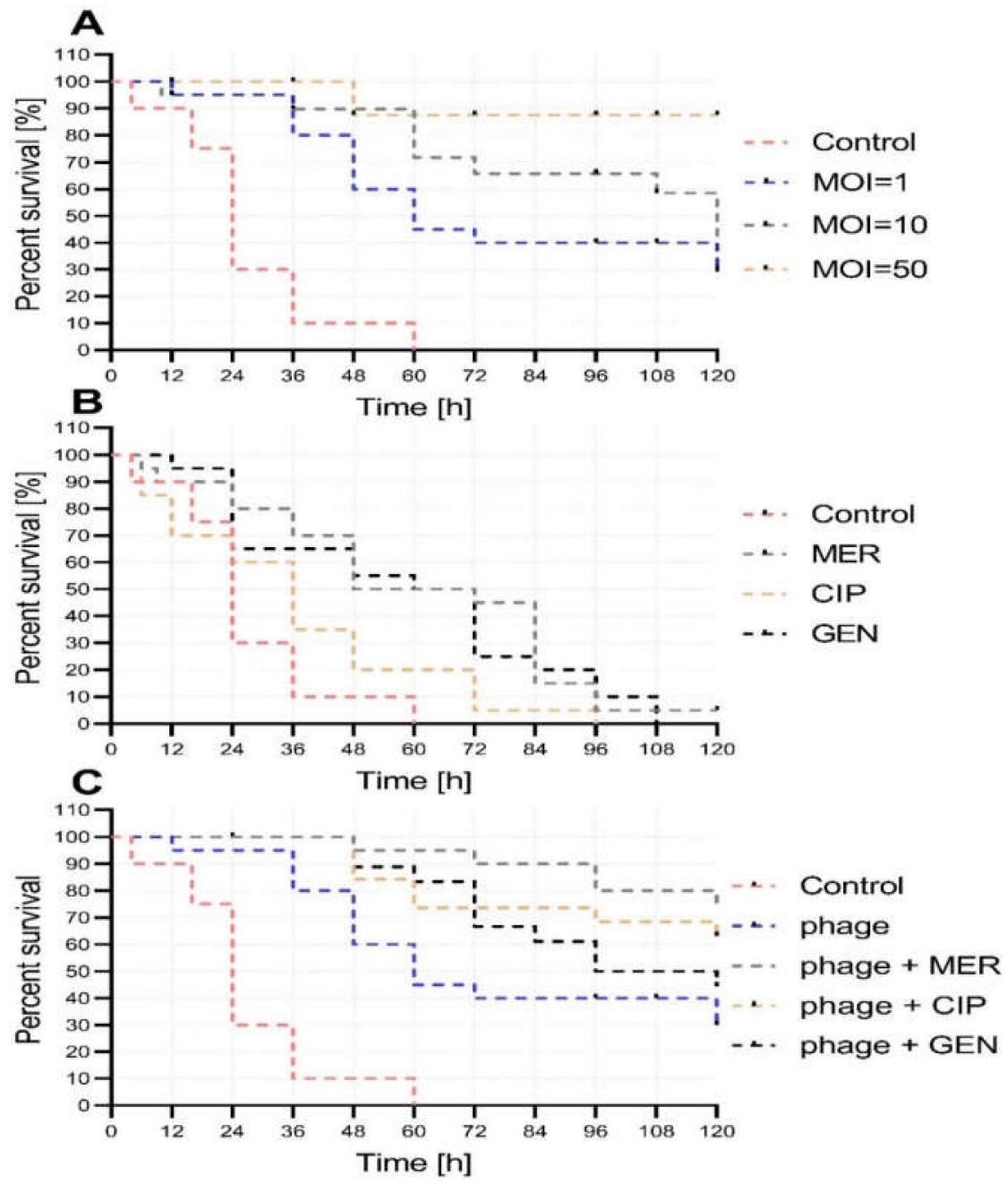

Figure 4. Survival of G. mellonella larvae. (A) Treatment of the A. baumannii (approx. $4 \times 10^{6} \mathrm{CFU}$ ) infected larvae at different MOIs of vB_AbaP_AGC01. (B) Analysis of the influence of the antibiotics meropenem (MER), ciprofloxacin (CIP), and gentamicin (GEN) on the survival of A. baumannii-infected larvae. (C) The activity of antibiotics (MER, CIP, and GEN) in combination with AGC01 phage $(\mathrm{MOI}=1)$ on survival of A. baumannii infected G. mellonella larvae. Survival of larvae treated with buffer, antibiotics, or phage alone was $100 \%$ (these lines were skipped for clarity). All experiments were technically repeated three times with triplicate biological replication. 


\section{Discussion}

Here, Acinetobacter baumannii infecting phage vB_AbaP_AGC01 was isolated, deeply characterized (phenotypically and genomically), and analyzed for antimicrobial activity in two infection models, namely HIP-B and G. mellonella larvae. Additionally, the influence of three different antibiotics (CIP, GEN, and MER) on phage activity in these models was determined. MER and CIP appeared to improve therapeutic outcomes of phage therapy in the in vivo model. The cumulative evidence supports the potential of vB_AbaP_AGC01 for therapeutic application alone or as a part of phage cocktails. Additionally, antibiotics (especially CIP and MER) improved therapeutic outcomes of phage therapy in in vivo model. However, to the best our knowledge this is the first study showing an antibiotic enhancement of the fully characterized lytic phage-based therapy against A. baumannii in human heat-inactivated plasma blood and Galleria mellonella model. The results suggest that the phages and antibiotics can act complementarily when administered together.

The vB_AbaP_AGC01 was classified (based on genome sequence similarity) as a member of the Friunavirus genus from a subfamily of Autographivirinae. Isolated AGC01 phage possesses a broad lytic spectrum and a high target specificity, highlighting its potential as a tool for phage therapy of aforementioned infections or as a component of a phage cocktail against A. baumannii [17,18]. The genome of the AGC01 was characterized and annotated, which meets the requirements of deep genome analysis of phages candidates for therapeutic purposes [19]. Additionally, the lytic spectrum of around 50\% suggests a broad host range of isolated phage. While, the host range of other Acinetobacter infecting phages varied between $2-68 \%$, and in some reports, newly isolated phages infect only their propagation host $[11,14,20]$. Additionally, the host range of acinetobacter-infecting podoviruses mainly depended on pectate lyase depolymerase domains located on the tail fibers [16,21-24]. It can be presumed that isolation and characterization of naturally occurring Acinetobacter phages is important and puts novel insights into their biology. Additionally, they could be used as a source of capsule degrading enzymes that also poses antimicrobial properties [22]. Production of the depolymerases could be related to bacteriophage-mediated biofilm disruption. Isolated phage is able to reduce biofilm biomass at all concentrations, and this suggests that AGC01 exhibits antibiofilm properties. During the phage-antibiotic combination therapy, phage-mediated biofilm disruption increased antibiotic penetration and antibiofilm activity [25]. Limited penetration of antibiotics throughout the biofilm is contributed to increased biofilm resistance to antibiotics.

Bacteriophages are very resilient to unfavorable environmental factors, such as temperature or $\mathrm{pH}$ changes [26,27]. In contrast, they are sensitive to divalent cation availability, which plays an essential role in the maintenance of the phage activity [28]. A decrease in the concentration of divalent metal ions, likely as a result of the EDTA chelation, was found to substantially restrict bacteriophage vB_AbaP_AGC01 activity when cations were not reconstructed (Supplementary Figure S2B). Similar findings were reported by Ma et al. (2018) [28], who observed that the lack of ion reconstruction decreased activity of bacteriophages infecting extraintestinal pathogenic E. coli strains in a HIP-B model. Additionally, phage antibacterial activity in HIP-B was suppressed by exogenous iron, magnesium, and calcium addition [28]. The present study illustrates the importance of divalent ions in determining phage activity in human blood and human blood-based sepsis models. Many other blood components, including hormones, lipids, carbohydrates, and metabolites, may also play a role in the determination of bacteriophage activity in blood.

To the best of our knowledge, to date, there is no report describing the activity of A. baumannii infecting phages combined with antibiotics in a human blood-based model. The present study demonstrates that AGC01 in combination with CIP and MER significantly decreases the bacterial cell load in such a model. The enhanced activity of bacteriophages combined with different classes of antibiotics in liquid bacterial culture has been previously described. The A. baumannii infecting phage KARL-1 combined with therapeutic doses of colistin significantly increases the clearance of bacterial cells [11]. In addition, MER augmented KARL-1 activity at a MOI $=10^{-7}$, which is similar to the manner in which AGC01 and MER in combination exhibit a synergistic increase in bacterial 
clearance. Blasco et al. (2019) observed that the synergistic effect of a Ab105-2 $\varphi \Delta C I$ lytic mutant combined with MER was maintained when $\frac{1}{4}$ MIC (minimal inhibitory concentration) and a MOI $=10$ were used [29]. Unfortunately, no synergistic effect was observed using KARL-1 in combination with CIP [11]. The combination of AGC01 with CIP resulted in similar enhancement of phage activity as was seen within combination with MER. The strain dependence of the PAS effect has been previously described by Pletezer et al. (2018) where the PAS effect was observed on $86.6 \%$ of Pseudomonas aeruginosa strains analyzed [30]. These variations observed suggest that the PAS effect may be a strain and/or phage dependent. Additionally, the medium or model used may also play an important role in this phenomenon. The PAS effect occurrence should be analyzed with the use of the in vivo and ex vivo models for each bacterial strain and phage respectively before the use of in vivo models.

The in vitro conditions used hitherto are not representative of animal infection models. Ideally, therapeutic evaluation of bacteriophages as candidates for phage therapy should include ex vivo and in vivo models. This motivated the evaluation of the antimicrobial activity of AGC01 and antibiotics in a greater wax moth larva (G. mellonella) model. G. mellonella was proposed as an alternative for the evaluation of the activity and toxicity of new antimicrobial agents [31]. Previous studies have shown the utility of this model in the assessment of phage therapy against Bordetella bronchiseptica, Cronobacter sakazakii, P. aeruginosa, and Burkholderia cepacia [19,32-35]. This model was also used for assessment of the phage therapy treatment against $A$. baumannii [35]. High MOI (100) single-dose phage $\mathrm{B} \varphi-\mathrm{R} 2096$ therapy increased the survival rates of larvae by approximately $50 \%, 24 \mathrm{~h}$ after $A$. baumannii infection [35]. We demonstrated that AGC01 treatment increases the survival rate of larvae from $30 \%$ to $90 \%$ at a MOI of 50, as well as observing a statistically significant increase in the survival rate of G. mellonella larvae treated with AGC01 at lower MOIs (1 and 10). The combination of phage with all used antibiotics was significantly more active than antibiotics used alone, and conversely, the combination of phage with CIP and MER statistically increased the effectiveness of phage therapy. MER was previously combined with bacteriophage KS12 infecting Burkholderia cepacia and the combined treatment resulted in a $20 \%$ decrease in mortality after $48 \mathrm{~h}$ and a $56 \%$ decrease after $72 \mathrm{~h}$ (when bacteria infected control group reached $100 \%$ mortality) [13]. Also, MER augmented KARL-1 activity in vitro-method-based approach but this study lacks in vivo analysis [11]. Importantly, after the injection of phages, antibiotics, or combinations of these agents, the melanization marker of the larva humoral response was not detected [36], which suggests that combined therapy based on bacteriophages and antibiotics may be both highly effective and safe. Increasements of the activity of phages combined with antibiotics might be promoted by bacterial cell stress response, which might promote phage replication inside of cell [11]. Synergistic action between antibiotics and bacteriophages might be extremely beneficial for infection treatment outcomes. Unfortunately, the actual knowledge of this phenomenon is limited and mostly speculative [37].

In conclusion, these results suggest that combined therapy poses the highest effectiveness, results should be considered with care. Especially, observed PAS effect is often strain-specific and results obtained with the use of the invertebrate models of $A$. baumannii infection need additional mammalian infection models for confirmation. Further investigations are necessary to understand and precise molecular mode of action of the phages-antibiotics combinations.

\section{Materials and Methods}

\subsection{Identification, Storage, and Growth of Bacterial Strains}

Acinetobacter baumannii (ATCC ${ }^{\circledR} 16909^{\mathrm{TM}}$ ) was obtained from ATCC (Manassas, VA, USA). In addition, 185 MDRAB strains isolated from clinical specimens were accessed at the Pomeranian Medical University in Szczecin (Szczecin, Poland). Strains were characterized using a VITEK ${ }^{\circledR} 2$ Compact Bacterial Identification System (bioMérieux, Warsaw, Poland) and validated by MALDI-TOF mass spectrometry. Antibiotic susceptibility was assessed according to CLSI guidance. Bacterial strains 
were stored at $-80^{\circ} \mathrm{C}$ before use. Bacterial strains were grown on blood agar (bioMérieux, Warsaw, Poland) for $24 \mathrm{~h}$ at $37^{\circ} \mathrm{C}$ to revitalize prior to further application.

\subsection{Bacteriophage Isolation, Purification, Propagation, and Host Range}

Bacteriophage was isolated from fishery pond sample (collected in Stargard, West Pomeranian Region, Poland) using A. baumannii $\mathrm{ATCC}^{\circledR} 16909^{\mathrm{TM}}$ as a host with the use of the standard enrichment technique [38]. After that, bacteriophage was triple time propagated from a single plaque for strain purification. Following the guidelines of Adriaenssens and Brister [39], isolated bacteriophage was designated vB_AbaP_AGC01 (also referred as AGC01). A high titer phage lysate was prepared by overnight propagation in a log-phase bacterial culture with shaking $(180 \mathrm{rpm})$ at $37^{\circ} \mathrm{C}$. The phage lysate was subsequently centrifuged at $2000 \times \mathrm{g}$ for $10 \mathrm{~min}$ at $4{ }^{\circ} \mathrm{C}$, and supernatant was filtered through a $0.2 \mu \mathrm{m}$ sterile filter (Sartorius Stedim Biotech, France).

The phage titer, and host range was determined by means of a double agar overlay or double layer agar (DLA) plaque assay as previously described [40]. A total of 185 clinical MDRAB strains, isolated from nosocomial infections in West Pomeranian and Lubusz regions of Poland, were used to assess the host range of the isolated bacteriophage. Host range analysis based on the efficacy of plating (EOP), allowed the exclusion of false-positive results [41]. The EOP was calculated according to Equation (1).

$$
\mathrm{EOP}=\frac{\text { average PFU on target bacteria }}{\text { average PFU on host bacteria }}
$$

\subsection{Bacteriophage Characterization}

Adsorption assay was performed as previously described [19]. The number of adsorbed phages was determined as a percent decrease in PFUs. The adsorption constants were calculated as previously recommended [42] following Equation (2).

$$
k=\frac{2.3}{\text { bacterial initial count }\left[{\left.\mathrm{CFU} \times \mathrm{mL}^{-1}\right] \times \text { time }[\mathrm{min}]}_{\log }\right.} \log _{10}\left(\frac{\text { phage initial counts }\left[\mathrm{PFU} \times \mathrm{mL}^{-1}\right]}{\text { phage count at time point }\left[\mathrm{PFU} \times \mathrm{mL}^{-1}\right]}\right)
$$

One-step growth, whereby isolated bacteriophages infect $A$. baumannii cells in a single cycle only, was performed, as described by Turner et al. [14] with minor modifications. Briefly, bacteriophages at a MOI of 0.05 were added to the bacterial culture and allowed to adsorb for $5 \mathrm{~min}$. Afterward, the sample was centrifuged at $13,000 \times g$, the supernatant was removed and the pellet was resuspended in equal to the initial volume of LB broth (A\&A Biotechnology, Poland). The sample was diluted to $10^{-7}$, incubated and titrated by triplicate overlay plaque assay to determine the single burst.

Bacteriophage lysis of liquid culture was evaluated as follows: Fresh LB medium supplemented with $10 \mathrm{mM} \mathrm{CaCl}_{2}$ (Sigma-Aldrich, Darmstadt, Germany) and $\mathrm{MgCl}_{2}$ (Sigma-Aldrich, Darmstadt, Germany) medium was inoculated using overnight $A$. baumannii cultures and was incubated shaking at $180 \mathrm{rpm}$ at $37^{\circ} \mathrm{C}$ to reach an optical density at $600 \mathrm{~nm}\left(\mathrm{OD}_{600 \mathrm{~nm}}\right)$ of 0.4 . At that point, bacterial cultures were treated with bacteriophage at MOIs of $0.1,0.25,0.5$. Samples were collected every $30 \mathrm{~min}$ after bacteriophage addition and the $\mathrm{OD}_{600 \mathrm{~nm}}$ was measured using an En-Vision 2105 multimode spectrophotometric plate reader (Perkin-Elmer, USA). Growth medium was used as the blank sample and bacterial culture with phage-free buffer and antibiotics added was used as growth control. The experiment was technically repeated three times, conducted in triplicate.

The thermal and $\mathrm{pH}$ stability of the AGC01 was conducted as previously described [43].

Phage vB_AbaP_AGC01 DNA was isolated with the use of the Genomic Mini AX Phage kit (A\&A Biotechnology, Poland). The complete genome sequence of AGC01 was determined in the DNA Sequencing and Oligonucleotide Synthesis Laboratory (oligo.pl) at the Institute of Biochemistry and Biophysics, Polish Academy of Sciences (Warsaw, Poland). The complete and fully annotated genome sequence of the $A$. baumannii phage vB_AbaP_AGC01 is available from the GenBank database (acc. no. MT263719). 
Prediction of open reading frames (ORFs) and genome annotations were performed with the use of Geneious Prime 2019.2.1 software (https://www.geneious.com). Genome annotations were verified by comparison of the related protein sequence with the use of blast software (blastp.ncbi.nlm.gov.pl). RNAP-based phylogeny was prepared with the use of MEGA X software [44]. Phage regulatory elements were scanned with the use of PHIRE software [45] and visualized using WebLogo [46]. The sequences of isolated bacteriophages were aligned and similarity was verified with the use of Circoletto software [47]. The bacteriophages vB_AbaP_AS11 (acc. no. NC_041915.1), IME200 (acc. no. KT804908.2), and FRI1 (acc. no. KR149290.1) were used for generation of sequence similarity graphs.

\subsection{Analysis of Bacterial Growth and Bacteriophage Activity in Blood Model}

All blood sample donors gave their informed consent for inclusion before they participated in the study. The study was conducted in accordance with the Declaration of Helsinki and the protocol was approved by the Local Ethics Committee (KB-0012/33/19).

Whole human blood was collected from healthy volunteers by means of a sterile syringe containing EDTA (S-Monovette ${ }^{\circledR}$, Sarstedt, Nümbrecht, Germany). Analysis of bacteriophage and antibiotic synergistic activity was performed in heat-inactivated plasma blood (HIP-B) according to Ma et al. (2018) [28] with minor modifications. HIP-B was prepared by centrifugation of the whole blood samples at $2000 \times g$ for $10 \mathrm{~min}$ and the plasma was heat-inactivated at $56^{\circ} \mathrm{C}$ for $1 \mathrm{~h}$. The pelleted erythrocytes and leukocytes were rinsed with sterile PBS buffer preheated to $37^{\circ} \mathrm{C}$. The heat-inactivated plasma was added to other blood components for blood reconstruction. For the reconstruction, $\mathrm{CaCl}_{2}$, $\mathrm{FeSO}_{4}$, or $\mathrm{MgCl}_{2}$ (all purchased from Sigma-Aldrich, Darmstadt, Germany) ions were added to a final concentration of $5 \mathrm{mM}$. Bacteriophage activity was measured in blood reconstructed with ions and in non-reconstructed blood.

To assess the growth rate of $A$. baumannii clinical strains in HIP-B, the blood sample was inoculated with $50 \mu \mathrm{L}$ of $A$. baumannii overnight culture and incubated for $24 \mathrm{~h}$ at $37^{\circ} \mathrm{C}$. Afterward, serial dilutions were prepared, plated on trypticase soy agar (TSA) (A\&A Biotechnology, Poland), and incubated for $24 \mathrm{~h}$ at $37^{\circ} \mathrm{C}$. Bacteriophage activity was assessed by inoculation of preheated HIP-B with A. baumannii at a final concentration of $1.5 \times 10^{5} \mathrm{CFU} \times \mathrm{mL}^{-1}$ and adding $\mathrm{vB}$ _AbaP_AGC01 at a MOI $=10$ before incubation at $37^{\circ} \mathrm{C}$. To assess the influence of antibiotics on phage activity, MER $\left(20 \mathrm{mg} \times \mathrm{L}^{-1}\right)$ (Sigma-Aldrich, Darmstadt, Germany), CIP $\left(10 \mathrm{mg} \times \mathrm{L}^{-1}\right)$ (Sigma-Aldrich, Darmstadt, Germany), and GEN ( $\left.8 \mathrm{mg} \times \mathrm{L}^{-1}\right)$ (Sigma-Aldrich, Darmstadt, Germany) were added to cultures as indicated. Samples were collected after $12 \mathrm{~h}$ of incubation at $37^{\circ} \mathrm{C}$. Serial dilutions were prepared of each experimental culture in PBS and these were plated on a TSA plate and incubated for $18-24 \mathrm{~h}$ at $37^{\circ} \mathrm{C}$. Bacterial colonies that grow in the presence of bacteriophage and bacteriophage/antibiotic combination were analyzed for phage sensitivity using the spot-test methodology.

\subsection{In Vivo Synergy in the Galleria Mellonella Model}

Only larvae exhibiting a uniform cream color and weighing $300 \mathrm{mg}$ (slight variations were used to calculate antibiotic dosage) were used for experiments. The in vivo test was performed as described by Szymczak et al. (2020) [19] with minor modifications. Briefly, G. mellonella larvae were infected with approximately $4 \times 10^{6} \mathrm{CFU}$ of $A$. baumannii into the larval hemolymph behind the last proleg. After $20 \mathrm{~min}$, the infected larvae were treated with the bacteriophage suspension at $\mathrm{MOI} \approx 1$, which was injected on the opposite side to the bacterial injection. Where antibiotics were used, these were premixed into the phage solution to the following final concentrations: $20 \mathrm{mg} \times \mathrm{kg}^{-1}$ MER, $10 \mathrm{mg}$ $\times \mathrm{kg}^{-1} \mathrm{CIP}$, or $8 \mathrm{mg} \times \mathrm{kg}^{-1} \mathrm{GEN}$. Doses were based on clinically relevant doses applied to human patients. Buffer, phage suspension, and antibiotic suspension controls were prepared when assessing bacteriophage toxicity. Larvae were incubated at $37^{\circ} \mathrm{C}$ and examined every $12 \mathrm{~h}$. The dark larvae that did not respond to physical contact were marked deceased. 


\subsection{Statistical Analysis}

Data were analyzed using one-way analysis of variance (ANOVA). $p$ values lower than 0.05 were considered statistically significant. Kaplan-Meier survival test was used for analysis of G. mellonella survival with the Mantel-Cox test. All statistical analyses were carried out using GraphPad Prism 7.04 software (Graph Pad Software, San Diego, CA, USA).

Supplementary Materials: Supplementary materials can be found at http://www.mdpi.com/1422-0067/21/12/4390/s1.

Author Contributions: Conceptualization, B.G.; methodology, B.G.; formal analysis, B.G., P.G.; investigation, B.G., M.R, P.G.; data curation, B.G.; writing—original draft preparation, B.G.; writing-review and editing, B.G., M.R., P.G., R.R., M.G., B.D., J.J.-K., D.Ś.-T., N.Ł., B.W.; visualization, B.G.; supervision, B.G., R.R., P.G.; project administration, B.G., R.R., B.D.; funding acquisition, B.G., R.R., B.D. All authors have read and agreed to the published version of the manuscript.

Funding: This research was funded by the National Science Centre (Poland) within the projects grant no. 2018/29/N/ST8/01043 (granted to B.G.), and no. 2018/31/B/ST8/03170 (granted to R.R.). Additionally, the project was partially financed from the program provided by the Polish Ministry of Science and Higher Education under the name "Regional Initiative of Excellence" in 2019-2022, project number 002/RID/2018/19, amount of financing 12.000.000 PLN. The APC was funded by Pomeranian Medical University (Poland).

Conflicts of Interest: The authors declare no conflict of interest. The funders had no role in the design of the study; in the collection, analyses, or interpretation of data; in the writing of the manuscript, or in the decision to publish the results.

\section{Abbreviations}

$\begin{array}{ll}\text { ANOVA } & \text { Analysis of variance } \\ \text { CFU } & \text { Colony-forming unit } \\ \text { CIP } & \text { Ciprofloxacin } \\ \text { CLSI } & \text { Clinical and Laboratory Standard Institute } \\ \text { DLA } & \text { Double layer agar } \\ \text { GEN } & \text { Gentamicin } \\ k & \text { Adsorption constant } \\ \text { LB } & \text { Luria Bertani broth } \\ \text { MALDI } & \text { Matrix-assisted laser desorption/ionization } \\ \text { MDRAB } & \text { Multidrug resistant Acinetobacter baumannii } \\ \text { MER } & \text { Meropenem } \\ \text { MOI } & \text { Multiplicity of infection } \\ \text { NCBI } & \text { National Center for Biotechnology Information } \\ \text { OD } & \text { Optical density } \\ \text { ORF } & \text { Open reading frame } \\ \text { PAS } & \text { Phage antibiotic synergy } \\ \text { PBS } & \text { Phosphate buffered saline } \\ \text { PFU } & \text { Plaque forming units } \\ \text { RNAP } & \text { RNA polymerase } \\ \text { SD } & \text { Standard deviation } \\ \text { TOF } & \text { Time-of-flight } \\ \text { TSA } & \text { Trypticase soy agar } \\ & \end{array}$

\section{References}

1. Lee, C.-R.; Lee, J.H.; Park, M.; Park, K.S.; Bae, I.K.; Kim, Y.B.; Cha, C.-J.; Jeong, B.C.; Lee, S.H. Biology of Acinetobacter baumannii: Pathogenesis, Antibiotic Resistance Mechanisms, and Prospective Treatment Options. Front. Cell. Infect. Microbiol. 2017, 7, 55. [CrossRef] [PubMed]

2. Espinal, P.; Vila, J.; López-Rojas, R.; Rolo, D.; Smani, Y.; Marti, S.; Lavigne, J.-P.; Pantel, A.; Pachón, J. Relationship Between Different Resistance Mechanisms and Virulence in Acinetobacter baumannii. Microb. Drug Resist. 2019, 25, 752-760. [CrossRef] [PubMed] 
3. Beceiro, A.; Moreno, A.; Fernandez, N.; Vallejo, J.A.; Aranda, J.; Adler, B.; Harper, M.; Boyce, J.D.; Bou, G. Biological cost of different mechanisms of colistin resistance and their impact on virulence in Acinetobacter baumannii. Antimicrob. Agents Chemother. 2014, 58, 518-526. [CrossRef] [PubMed]

4. World Health Organization. Global Priority List of Antibiotic-Resistant Bacteria to Guide Research, Discovery, and Development of New Antibiotics; World Health Organization: Geneva, Switzerland, 2017.

5. Antunes, L.C.S.; Visca, P.; Towner, K.J. Acinetobacter baumannii: Evolution of a global pathogen. Pathog. Dis. 2014, 71, 292-301. [CrossRef]

6. Potron, A.; Poirel, L.; Nordmann, P. Emerging broad-spectrum resistance in Pseudomonas aeruginosa and Acinetobacter baumannii: Mechanisms and epidemiology. Int. J. Antimicrob. Agents 2015, 45, 568-585. [CrossRef]

7. Kropinski, A.M. Bacteriophage research—What we have learnt and what still needs to be addressed. Res. Microbiol. 2018, 169, 481-487. [CrossRef]

8. Clokie, M.R.; Millard, A.D.; Letarov, A.V.; Heaphy, S. Phages in nature. Bacteriophage 2011, 1, 31-45. [CrossRef]

9. Bruttin, A.; Brüssow, H. Human volunteers receiving Escherichia coli phage T4 orally: A safety test of phage therapy. Antimicrob. Agents Chemother. 2005, 49, 2874-2878. [CrossRef]

10. Turner, D.; Ackermann, H.W.; Kropinski, A.M.; Lavigne, R.; Sutton, J.M.; Reynolds, D.M. Comparative analysis of 37 Acinetobacter bacteriophages. Viruses 2018, 10, 5. [CrossRef]

11. Jansen, M.; Wahida, A.; Latz, S.; Krüttgen, A.; Häfner, H.; Buhl, E.M.; Ritter, K.; Horz, H.-P. Enhanced antibacterial effect of the novel T4-like bacteriophage KARL-1 in combination with antibiotics against multi-drug resistant Acinetobacter baumannii. Sci. Rep. 2018, 8, 14140. [CrossRef]

12. Łoś, J.M.; Golec, P.; Wẹgrzyn, G.; Wẹgrzyn, A.; Łoś, M. Simple method for plating Escherichia coli bacteriophages forming very small plaques or no plaques under standard conditions. Appl. Environ. Microbiol. 2008, 74, 5113-5120. [CrossRef] [PubMed]

13. Kamal, F.; Dennis, J.J. Burkholderia cepacia complex phage-antibiotic synergy (PAS): Antibiotics stimulate lytic phage activity. Appl. Environ. Microbiol. 2015, 81, 1132-1138. [CrossRef] [PubMed]

14. Turner, D.; Wand, M.E.; Briers, Y.; Lavigne, R.; Sutton, J.M.; Reynolds, D.M. Characterisation and genome sequence of the lytic Acinetobacter baumannii bacteriophage vB_AbaS_Loki. PLoS ONE 2017, 12, e0172303. [CrossRef] [PubMed]

15. Farrugia, D.N.; Elbourne, L.D.H.; Hassan, K.A.; Eijkelkamp, B.A.; Tetu, S.G.; Brown, M.H.; Shah, B.S.; Peleg, A.Y.; Mabbutt, B.C.; Paulsen, I.T. The Complete Genome and Phenome of a Community-Acquired Acinetobacter baumannii. PLoS ONE 2013, 8, e58628. [CrossRef]

16. Hernandez-Morales, A.C.; Lessor, L.L.; Wood, T.L.; Migl, D.; Mijalis, E.M.; Cahill, J.; Russell, W.K.; Young, R.F.; Gill, J.J. Genomic and Biochemical Characterization of Acinetobacter Podophage Petty Reveals a Novel Lysis Mechanism and Tail-Associated Depolymerase Activity. J. Virol. 2018, 92, e01064-17. [CrossRef]

17. Łubowska, N.; Grygorcewicz, B.; Kosznik-Kwaśnicka, K.; Zauszkiewicz-Pawlak, A.; Węgrzyn, A.; Dołegowska, B.; Piechowicz, L. Characterization of the Three New Kayviruses and Their Lytic Activity Against Multidrug-Resistant Staphylococcus aureus. Microorganisms 2019, 7, 471. [CrossRef]

18. Melo, L.D.R.; Brandão, A.; Akturk, E.; Santos, S.B.; Azeredo, J. Characterization of a new Staphylococcus aureus Kayvirus harboring a lysin active against biofilms. Viruses 2018, 10, 182. [CrossRef]

19. Szymczak, M.; Grygorcewicz, B.; Karczewska-Golec, J.; Decewicz, P.; Pankowski, J.A.; Országh-Szturo, H.; Bącal, P.; Dołęgowska, B.; Golec, P. Characterization of a Unique Bordetella bronchiseptica vB_BbrP_BB8 Bacteriophage and Its Application as an Antibacterial Agent. Int. J. Mol. Sci. 2020, 21, 1403. [CrossRef]

20. Yang, H.; Liang, L.; Lin, S.; Jia, S. Isolation and characterization of a virulent bacteriophage AB1 of Acinetobacter baumannii. BMC Microbiol. 2010, 10, 131. [CrossRef]

21. Lai, M.J.; Chang, K.C.; Huang, S.W.; Luo, C.H.; Chiou, P.Y.; Wu, C.C.; Lin, N.T. The tail associated protein of Acinetobacter baumannii phage qab6 is the host specificity determinant possessing exopolysaccharide depolymerase activity. PLOS ONE 2016, 11, e0153361. [CrossRef]

22. Liu, Y.; Mi, Z.; Mi, L.; Huang, Y.; Li, P.; Liu, H.; Yuan, X.; Niu, W.; Jiang, N.; Bai, C.; et al. Identification and characterization of capsule depolymerase Dpo48 from Acinetobacter baumannii phage IME200. PeerJ 2019, 7, e6173. [CrossRef] [PubMed]

23. Domingo-Calap, P.; Beamud, B.; Mora-Quilis, L.; González-Candelas, F.; Sanjuán, R. Isolation and Characterization of Two Klebsiella pneumoniae Phages Encoding Divergent Depolymerases. Int. J. Mol. Sci. 2020, 21, 3160. [CrossRef] [PubMed] 
24. Oliveira, H.; Costa, A.R.; Konstantinides, N.; Ferreira, A.; Akturk, E.; Sillankorva, S.; Nemec, A.; Shneider, M.; Dötsch, A.; Azeredo, J. Ability of phages to infect Acinetobacter calcoaceticus-Acinetobacter baumannii complex species through acquisition of different pectate lyase depolymerase domains. Environ. Microbiol. 2017, 19, 5060-5077. [CrossRef] [PubMed]

25. Bedi, M.S.; Verma, V.; Chhibber, S. Amoxicillin and specific bacteriophage can be used together for eradication of biofilm of Klebsiella pneumoniae B5055. World J. Microbiol. Biotechnol. 2009, 25, 1145-1151. [CrossRef]

26. Jończyk, E.; Kłak, M.; Międzybrodzki, R.; Górski, A. The influence of external factors on bacteriophages-Review. Folia Microbiol. (Praha) 2011, 56, 191-200. [CrossRef] [PubMed]

27. Grygorcewicz, B.; Grudziński, M.; Wasak, A.; Augustyniak, A.; Pietruszka, A.; Nawrotek, P. Bacteriophage-mediated reduction of Salmonella Enteritidis in swine slurry. Appl. Soil Ecol. 2017, 119, 179-182. [CrossRef]

28. Ma, L.; Green, S.I.; Trautner, B.W.; Ramig, R.F.; Maresso, A.W. Metals Enhance the Killing of Bacteria by Bacteriophage in Human Blood. Sci. Rep. 2018, 8, 2326. [CrossRef]

29. Blasco, L.; Ambroa, A.; Lopez, M.; Fernandez-Garcia, L.; Bleriot, I.; Trastoy, R.; Ramos-Vivas, J.; Coenye, T.; Fernandez-Cuenca, F.; Vila, J.; et al. Combined use of the Ab105-2 $\phi \Delta C I$ lytic mutant phage and different antibiotics in clinical isolates of multi-resistant Acinetobacter baumannii. Microorganisms 2019, 7, 556. [CrossRef]

30. Pletzer, D.; Mansour, S.C.; Hancock, R.E.W. Synergy between conventional antibiotics and anti-biofilm peptides in a murine, sub-cutaneous abscess model caused by recalcitrant ESKAPE pathogens. PLoS Pathog. 2018, 14, e1007084. [CrossRef]

31. Desbois, A.P.; Coote, P.J. Wax moth larva (Galleria mellonella): An in vivo model for assessing the efficacy of antistaphylococcal agents. J. Antimicrob. Chemother. 2011, 66, 1785-1790. [CrossRef]

32. Seed, K.D.; Dennis, J.J. Experimental bacteriophage therapy increases survival of Galleria mellonella larvae infected with clinically relevant strains of the Burkholderia cepacia complex. Antimicrob. Agents Chemother. 2009, 53, 2205-2208. [CrossRef] [PubMed]

33. Abbasifar, R.; Kropinski, A.M.; Sabour, P.M.; Chambers, J.R.; MacKinnon, J.; Malig, T.; Griffiths, M.W. Efficiency of bacteriophage therapy against Cronobacter sakazakii in Galleria mellonella (greater wax moth) larvae. Arch. Virol. 2014, 159, 2253-2261. [CrossRef] [PubMed]

34. Beeton, M.L.L.; Alves, D.R.R.; Enright, M.C.C.; Jenkins, A.T.A.T.A. Assessing phage therapy against Pseudomonas aeruginosa using a Galleria mellonella infection model. Int. J. Antimicrob. Agents 2015, 46, 196-200. [CrossRef]

35. Jeon, J.; Park, J.H.; Yong, D. Efficacy of bacteriophage treatment against carbapenem-resistant Acinetobacter baumannii in Galleria mellonella larvae and a mouse model of acute pneumonia. BMC Microbiol. 2019, 19, 70. [CrossRef]

36. Tsai, C.J.Y.; Loh, J.M.S.; Proft, T. Galleria mellonella infection models for the study of bacterial diseases and for antimicrobial drug testing. Virulence 2016, 5594, 214-229. [CrossRef] [PubMed]

37. Segall, A.M.; Roach, D.R.; Strathdee, S.A. Stronger together? Perspectives on phage-antibiotic synergy in clinical applications of phage therapy. Curr. Opin. Microbiol. 2019, 51, 46-50. [CrossRef]

38. Twest, R.; Kropinski, A.M.; Van Twest, R.; Kropinski, A.M. Bacteriophage enrichment from water and soil. Methods Mol. Biol. 2009, 501, 15-21.

39. Adriaenssens, E.M.; Rodney Brister, J. How to name and classify your phage: An informal guide. Viruses 2017, 9, 70. [CrossRef]

40. Mazzocco, A.; Waddell, T.E.; Lingohr, E.; Johnson, R.P. Enumeration of Bacteriophages by the Direct Plating Plaque Assay. In Bacteriophages; Humana Press: Totowa, NJ, SAD, 2009; Volume 501, ISBN 978-1-58829-682-5.

41. Mirzaei, M.K.; Nilsson, A.S. Isolation of phages for phage therapy: A comparison of spot tests and efficiency of plating analyses for determination of host range and efficacy. PLoS ONE 2015, 10, e0118557. [CrossRef]

42. Kropinski, A.M. Measurement of the rate of attachment of bacteriophage to cells. Methods Mol. Biol. 2009, 501, 151-155.

43. Grygorcewicz, B.; Chajecka-Wierzchowska, W.; Augustyniak, A.; Wasak, A.; Stachurska, X.; Nawrotek, P.; Dołegowska, B. In-milk inactivation of Escherichia coli O157: H7 by the environmental lytic bacteriophage ECPS-6. J. Food Saf. 2019, 40, e12747. [CrossRef]

44. Kumar, S.; Stecher, G.; Li, M.; Knyaz, C.; Tamura, K. MEGA X: Molecular Evolutionary Genetics Analysis across Computing Platforms. Mol. Boil. Evol. 2018, 35, 1547-1549. [CrossRef] [PubMed] 
45. Lavigne, R.; Sun, W.D.; Volckaert, G. PHIRE, a deterministic approach to reveal regulatory elements in bacteriophage genomes. Bioinformatics 2004, 20, 629-635. [CrossRef] [PubMed]

46. Crooks, G.E.; Hon, G.; Chandonia, J.M.; Brenner, S.E. WebLogo: A sequence logo generator. Genome Res. 2004, 14, 1188-1190. [CrossRef]

47. Darzentas, N. Circoletto: Visualizing sequence similarity with Circos. Bioinformatics 2010, 26, $2620-2621$. [CrossRef] 